\title{
Study of the electronic and magnetic properties of the CoRhMnSn quaternary Heusler alloy
}

\section{Ilhem Benaisti}

Laboratoire de l'Energie et des Systèmes Intelligents, Faculty of Science and Technology, University

Djilali Bounaama, Khemis Miliana 44225, Algeria

Nacir Guechi

Laboratoire d'Etudes des Surfaces et Interfaces des Matériaux Solides, University Ferhat Abbas Setif 1, Setif 19000, Algeria https://orcid.org/0000-0001-9328-8362

Mourad Dehbaoui ( $D$ mourad.dehbaoui@gmail.com )

Laboratoire de Physique des Techniques Expérimentales et ses Applications, Université de Médéa 26000,Algeria https://orcid.org/0000-0001-8681-5099

\section{Abdelali Boukaoud}

Laboratoire de Physique des Techniques Expérimentales et ses Applications, Université de Médéa 26000,Algeria

\section{Research Article}

Keywords: Spintronic, Heusler alloy, Ferromagnetism, DFT

Posted Date: February 9th, 2021

DOI: https://doi.org/10.21203/rs.3.rs-215061/v1

License: (c) (i) This work is licensed under a Creative Commons Attribution 4.0 International License.

Read Full License 


\title{
Study of the electronic and magnetic properties of the CoRhMnSn quaternary Heusler alloy
}

\author{
Ilhem Benaisti $^{1}$, Nacir Guechi ${ }^{2}$, Mourad Dehbaoui $^{3}$, Abdelali Boukaoud ${ }^{3}$ \\ ${ }^{1}$ Laboratoire de l'Energie et des Systèmes Intelligents, Faculty of Science and Technology, University \\ Djilali Bounaama, Khemis Miliana 44225, Algeria. \\ ${ }^{2}$ Laboratoire d'Etudes des Surfaces et Interfaces des Matériaux Solides, University Ferhat Abbas Setif 1, \\ Setif 19000, Algeria. \\ ${ }^{3}$ Laboratoire de Physique des Techniques Expérimentales et ses Applications, Université de Médéa \\ 26000, Algeria.
}

*Corresponding author at: LPTEA, Université de Médéa, Bloc des laboratoires de recherche, Ain Dheb Médéa 26000 Algérie (M. Dehbaoui).

ORCID: 0000-0001-8681-5099

E-mail address: mourad.dehbaoui@gmail.com

dehbaoui.mourad@univ-medea.dz 


\section{Introduction}

Electronic devices based on the integration of component like semiconductors, metals and insulators have been subject of research and development over decades. In conventional electronic devices the Information is processed exclusively by manipulating the charge of the electrons. The arising field of spintronics deals, in addition to the electron charge, with the spin of electrons in a metallic or semiconducting systems. The spintronic uses the spin and the charge, of electrons to manipulate the information in new promising devices. Among the proposed materials to achieve such performance, we have the Heusler alloys. They are an intermetallic compound with interesting properties, consisting of $3 \mathrm{~d}$ transition elements and some nonmagnetic element from main group. Since their discovery in 1903 [1], Heusler alloys doesn't stop having interest of research all over the word. The Heusler alloys could have semiconducting, metallic, magnetic or nonmagnetic behaviour. The material exhibits a strong sensitivity to the magnetic field and pressure, as well as it constitutes an important media for generation of spin currents. The very recent discovery of Spin Gapless Semiconductors (SGS) [2] in Heusler structures led to more prospect and more interest after material for new potential applications using magneto-electronics, thermoelectric and optoelectronics phenomena.

Typically, Heusler alloys are crystalline materials composed of two or three transition metal elements (labelled X, X' and Y) and one non-magnetic element (labelled Z) from 13-17 group. Heusler alloys can be divided into three categories: $(a)$ half-Heuslers with XYZ chemical formula; $(b)$ full-Heuslers with $\mathrm{X}_{2} \mathrm{YZ}$ chemical formula; and $(c)$ quaternary-Heuslers with $\mathrm{XX}$ 'YZ chemical formula [3-6]. These alloys have attracted the attention of many scientific researchers due to their remarkable physical properties, including a high spin polarization, high curie temperatures and a half-metallicity with a ferromagnetic ground state, [7-8]. For these reasons, the Heusler alloys are identified as potential candidates for spintronics applications, such as giant magneto-resistance devices, spin injection, magnetic tunnel junctions, and shape memory devices [9-11].

Recently, quaternary Heusler compounds containing Mn atom, such as the CoRhMnSn quaternary Heusler alloy, have attracted significant interest because of their noteworthy magneto-electronic properties [12]. In this work, we present and discuss the structural and geometry optimization of a quaternary Heusler compound the CoRhMnSn. Using accurate first-principles calculations, the hydrostatic pressure effect on the electronic and magnetic properties of the proposed Heusler alloy is also investigated.

\subsection{Computational methods}

The electronic band structures and structural calculations were performed using first principle calculations within the framework of the density functional theory (DFT) [13].In order to solving the Kohn-Sham equations, we have used the pseudopotential plane-wave (PP-PW) method as implemented in the Cambridge Serial Total-Energy Package (CASTEP) [14]. The exchangecorrelation potential was parameterized using the generalized gradient approximation GGAPBEsol[15], which has been specially developed to improve the exchange-correlation effect in solid materials. The ultrasoft pseudopotential approach [16] was used to model the interaction potential between the nucleus, frozen and valence electrons. The considered valence states are: Co: $3 \mathrm{~d}^{7} 4 \mathrm{~s}^{2}$, Rh: $4 \mathrm{~d}^{8} 5 \mathrm{~s}^{1}, \mathrm{Mn}: 3 \mathrm{~d}^{5} 4 \mathrm{~s}^{2}, \mathrm{Sn}: 4 \mathrm{~s}^{2} 4 \mathrm{p}^{2}$. A plane-wave basis set cut-off energy of $400 \mathrm{eV}$ and a $12 \times 12 \times 12 \mathrm{k}$ points [17] grid for the integration over the Brillouin zone (BZ) were selected to guarantee sufficiently accurate total energy calculations. The geometry optimization was performed at each fixed pressure with the following convergence criteria: $(i)$ the total energy of the system was smaller 
than $5 \times 10^{-6} \mathrm{eV} /$ atom; (ii) the maximum stress was smaller than $0.02 \mathrm{GPa}$; (iii) the displacement of atoms was less than $5 \times 10^{-4} \AA$.

\section{Results and discussion}

The magneto-electronic properties of Heusler alloys are strongly dependent on the atomic arrangements in the crystal structure [3-4]. The equiatomic quaternary Heusler alloys XX'YZ crystallize in the cubic structure LiMgPdSb-type denoted as Y-type with space group no. 216. This structure type exhibits a primitive unit cell containing four atoms in the following Wyckoff positions: $4 \mathrm{a}(0,0,0), 4 \mathrm{~b}(1 / 2,1 / 2,1 / 2), 4 \mathrm{c}(1 / 4,1 / 4,1 / 4)$, and $4 \mathrm{~d}(3 / 4,3 / 4,3 / 4)$ [18]. The structure consists of four interpenetrating fcc sublattice. The atoms on Wyckoff positions $4 \mathrm{a}$ and $4 \mathrm{c}$ form a $\mathrm{ZnS}$ type sublattice, the atoms on Wyckoff positions 4 band $4 \mathrm{~d}$ form a second $\mathrm{ZnS}$-type sublattice with atoms in $4 \mathrm{~b}$ occupying the octahedral sites (Figure1).

Based on the atomic arrangements, there are three possible non-equivalent site occupancies within the considered structures. They are denoted as: Type-I, Type-II, Type-III as shown in Fig. 1.The corresponding positions for each atomic arrangement are summarized in Table1.

Table 1. here. Ilhem et al...

Figure 1. here. Ilhem et al...

In order to find the ground state crystal structure of CoRhMnSn, a geometry optimization was performed by computing the total energy of the unit cell at each fixed pressure for the three structure types $\left(\mathrm{Y}_{1}, \mathrm{Y}_{2}\right.$ and $\left.\mathrm{Y}_{3}\right)$ with non-magnetic $(\mathrm{NM})$ and ferromagnetic (FM) states. The calculated total energies using GGA-PBEsol versus unit-cell volumes have been fitted to the BirchMurnaghan equation of state (Eq. 1) [19] and are displayed in Fig. 2. From the sketched curves, one can easily note that the lowest energy belongs to the FM state in the $\mathrm{Y}_{1}$-type structure. Clearly, the $\mathrm{Y}_{1}$-type arrangement forms the ground state structure of for CoRhMnSn compound, and hence, we will discuss the magneto-electronic properties related to this atomic configuration only. Note that, for $\mathrm{Y}_{1}-\mathrm{CoRhMnSn}$ structure, the $\mathrm{Sn}$ and $\mathrm{Mn}$ atoms occupy the octahedral sites while Co and $\mathrm{Rh}$ atoms occupy the tetrahedral ones.The result emphasizes on one hand, a covalent bonding interaction between $\mathrm{Sn}$ and $\mathrm{Mn}$ elements which plays a major role for the electronic properties of the material. On the other hand, it is worth mentioning that the Co and $\mathrm{Rh}$ atoms built a NaCl-type sublattice, leading to a strong ionic character interaction between $\mathrm{Co}$ and $\mathrm{Rh}$ elements.

$$
E=E_{0}+\left(\frac{9}{16}\right) * B_{0} * V_{0} *\left(\left(B^{\prime}-4\right) *\left(\frac{V}{V_{0}}\right)^{\frac{-2}{3}}-B^{\prime}+6\right) *\left(\left(\frac{V}{V_{0}}\right)^{\frac{-2}{3}}-1\right)^{2}
$$

Figure2.here. Ilhem et al... 
The lowest total energy per unit cell $\left(E_{0}\right)$, equilibrium lattice constant $\left(a_{0}\right)$, bulk modulus $\left(B_{0}\right)$ and its derivative $\left(B^{\prime}=d B / d V\right)$ are listed in Table 2 for the $\mathrm{Y}_{1}-\mathrm{CoRhMnSn}$ crystal structure. It is important to note thatthe calculated results are in good agreement with the available experimental and theoretical data.

Table 1. here. Ilhem et al...

The electronic band structure analysis is essential to understand the physical properties of solid materials. The $\mathrm{Y}_{1}$-CoRhMnSnband structures are sketched in Fig. 3, at three different pressures -16, 0 and 35GPa. From Fig. 3, one observes that the spin majority (spin up) and the spin minority (spin down) states exhibit metallic natures at $-16 \mathrm{GPa}$ and $0 \mathrm{GPa}$, whereas, they shows a different behaviour at $35 \mathrm{GPa}$. At $35 \mathrm{GPa}$, the spin majority bands maintain its metallic behaviour with a nonzero density of states at Fermi level $\left(\mathrm{E}_{\mathrm{F}}\right)$, while the spin minority bands show a semiconducting nature with an indirect gap of $0.17 \mathrm{eV}$ along with $\Gamma-\mathrm{X}$ direction. For the spin minority bands, the valence band maximum (VBM) is located at $\Gamma$-high symmetry point, and the conduction band minimum $(\mathrm{CBM})$ is situated at $\mathrm{X}$-high symmetry point. As a result, and since it has a spin polarization equal to $100 \%$ at Fermi level, the CoRhMnSn compound can be considered as a perfect half-metallic alloy at $35 \mathrm{GPa}$.

Figure3.here. Ilhem et al...

The variations of the VBM and CBM as functions of hydrostatic pressure from -20 GPa to 50GPa for the spin minority states is shown in Fig. 4. It is worth noting that the variation of pressure from -20 $\mathrm{GPa}$ to 50GPa does not affect the spin majority bands; the CoRhMnSn compound behaves like a metal along this spin channel. Furthermore, from Fig. 4, we can see that both VBM and CBM decreases with the increasing pressure, and hence we can deduce the following statements: (i) for pressure values less than $35 \mathrm{GPa}$, the studied compound has a metallic nature, (ii) for pressures around $35 \mathrm{GPa}$, the CoRhMnSn compound has a nearly half metallic nature, (iii) At 35GPa, the CoRhMnSn has a perfect half metallic behaviour, and (iv) for pressures greater than $35 \mathrm{GPa}$, the CoRhMnSn maintain its acquired half-metallic character.

Figure4.here. Ilhem et al...

For quaternary Heusler alloys (XX'YZ), the origin of the half-metallic gap is the sequence of the strong hybridization between the $d$ states around the Fermi level of the transition metal atoms (X, $\mathrm{X}^{\prime}$ and Y).The $s$ and $p$ orbitals of the $\mathrm{Z}$ atom are commonly lying far below Fermi level and do not contribute to the half-metallic gap formation [10-20-21]. For CoRhMnSn, the $d$-Co states hybridize with the $d$-Rh states and create the $2 \mathrm{e}_{\mathrm{g}}$ and $3 \mathrm{t}_{2 \mathrm{~g}}$ bonding hybrid orbitals. These orbitals hybridize again

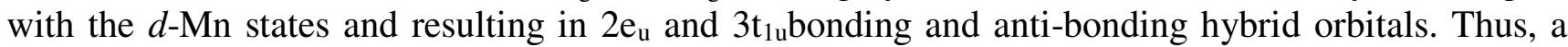
gap appears for the spin minority channel at $35 \mathrm{GPa}$ due to the separations between $2 \mathrm{e}_{\mathrm{u}}$ and $3 \mathrm{t}_{1 \mathrm{u}}$ states (see Fig. 5). Note that, along the spin-up direction, the total number of energy levels and symmetry irreducible representations are identical to those along the spin-down direction. 
Figure5.here. Ilhem et al...

In Figure 6, we display the pressure effect on the total and atomic magnetic moments of the CoRhMnSn Heusler alloy. It is known that for a perfect half metallic material, the total magnetic moment per unit cell is an integer value and obeys to the Slater-Pauling rule [22-23]. The calculated value of the magnetic moment of CoRhMnSn is equal to $5 \mu_{\square \square}$ which agree very well with the " $\mathrm{M}_{\mathrm{t}}=\mathrm{Z}_{\mathrm{t}}$ " 24" Slater Pauling rule. For CoRhMnSn, the spin minority bands contain 12 electrons and the total number of valence electrons per unit cell $\left(Z_{t}\right)$ is equal to 29. From Fig. 6, we see also that the variation of pressure does not affect the total magnetic moment of the CoRhMnSn compound. Actually, with the increasing pressure, the magnetic moment of the Mn atom decreases, whereas those of Co and $\mathrm{Rh}$ atoms increase, leading to an invariant total magnetic moment under pressure variations.

Figure6.here. Ilhem et al...

\section{Conclusion}

In this work, the electronic and magnetic properties of the CoRhMnSn quaternary Heusler alloy were investigated using the pseudopotential plane-wave (PP-PW) method with the generalized gradient approximation (GGA-PBEsol). Regarding to the magnetic sensitivity of the material, the two spin states were considered during calculation. The equilibrium total energy was found to correspond to $Y$ type $I$ atomic arrangements, indicating a covalent bonding interaction between $\mathrm{Sn}$ and $\mathrm{Mn}$ elements and hence playing a major role for the electronic properties of the material. According to this $Y$-type I arrangement there is also indication of a strong ionic character interaction between Co and $\mathrm{Rh}$ elements. Precisely, in this paper, it is first to report the effect of the hydrostatic pressure on the structural and magnetic properties of CoRhMnSn quaternary Heusler alloy. It was found that the intensity of the density of state (DOS) at the Fermi level and the width of energy gap can be adjusted by pressure variations. The magnetic property analysis through the total magnetic calculation, revealed that at $35 \mathrm{GPa}$, the CoRhMnSn compound is found to be a perfect half metallic ferromagnetic material with a net total magnetic moment of $5 \mu_{\mathrm{B}}$. The hydrostatic pressure variation analysis demonstrated that the total magnetic moment of the alloy conserves the same value of $5 \mu_{\mathrm{B}}$, for all electronic compound behaviours. It is worth mentioning that, the steady magnetic moment and the half metallic characters of the studied alloy, endorse the idea of considering the CoRhMnSn compound as a good candidate for a potential use for example as a spin channel directional control of current in spintronic devices. 


\section{References}

1. F. Heusler, Übermagnetischemanganlegierungen. Verhandlungen der Deutschen Physikalischen Gesellschaft, 5, 219. (1903)

2. S. Ouardi, G. H. Fecher, C. Felser, Kübler J., Realization of spin gapless semiconductors: The Heusler compound Mn 2 CoAl. Physical review letters, 110(10), 100401. (2013)

3. C. Felser, A. Hirohata, Heusler alloys. (Springer, Berlin, 2015)

4. T. Graf, C. Felser, S. S.Parkin, Simple rules for the understanding of Heusler compounds. Progress in solid state chemistry 39(1): 1-50. (2011)

5. R. A. De Groot, F. M. Mueller, P. G. Van Engen, K. H. J. Buschow, New class of materials: half-metallic ferromagnets. Physical Review Letters, 50(25), 2024. (1983)

6. J. Kübler, A. R. William, C. B. Sommers, Formation and coupling of magnetic moments in Heusler alloys. Physical Review B, 28(4), 1745. (1983)

7. C. J. Palmstrøm, Heusler compounds and spintronics. Progress in Crystal Growth and Characterization of Materials, 62(2), 371-397. (2016)

8. V. Alijani, J. Winterlik, G. H. Fecher, S. S. Naghavi, C. Felser, Quaternary half-metallic Heusler ferromagnets for spintronics applications. Physical Review B, 83(18), 184428. (2011)

9. T. Graf, S. S. Parkin, C. Felser, Heusler compounds: A material class with exceptional properties. IEEE Transactions on Magnetics, 47(2), 367-373. (2010)

10. L. Bainsla, K. G. Suresh, Equiatomic quaternary Heusler alloys: A material perspective for spintronic applications. Applied Physics Reviews, 3(3), 031101. (2016)

11. C. Felser, G. H. Fecher, B. Balke, Spintronics: a challenge for materials science and solid-state chemistry. AngewandteChemie International Edition, 46(5), 668-699. (2007)

12. V. Alijani, J. Winterlik, G. H. Fecher, S. S. Naghavi, S. Chadov, T. Gruhn, C. Felser, Quaternary Heusler compounds $\mathrm{Co}_{2-\mathrm{x}} \mathrm{Rh}_{\mathrm{x}} \mathrm{MnZ}(\mathrm{Z}=\mathrm{Ga}, \mathrm{Sn}, \mathrm{Sb})$ : crystal structure, electronic structure, and magnetic properties. Journal of Physics: Condensed Matter, 24(4), 046001. (2012)

13. P. Hohenberg, W. Kohn, Density Functional Theory (DFT). Phys. Rev, 136, B864. (1964)

14. M. C. Payne, M. P. Teter, D. C. Allan, T. A. Arias, A. J. Joannopoulos, Iterative minimization techniques for ab initio total-energy calculations: molecular dynamics and conjugate gradients. Reviews of modern physics, 64(4), 1045. (1992)

15. J. P. Perdew, K. Burke, M. Ernzerhof, Generalized gradient approximation made simple. Physical review letters, 77(18), 3865. (1996)

16. K. Laasonen, R. Car, C. Lee, D. Vanderbilt, Implementation of ultrasoft pseudopotentials in ab initio molecular dynamics. Physical Review B, 43(8), 6796. (1991)

17. H. J. Monkhorst, J. D. Pack, Special points for Brillouin-zone integrations. Physical review B, 13(12), 5188. (1976)

18. G. E. Bacon, J. S. Plant, Chemical ordering in Heusler alloys with the general formula $\mathrm{A}_{2} \mathrm{BC}$ or ABC. Journal of Physics F: Metal Physics, 1(4), 524. (1971)

19. F. Birch, The effect of pressure upon the elastic parameters of isotropic solids, according to Murnaghan's theory of finite strain. Journal of Applied Physics, 9(4), 279-288. (1938)

20. G. Z. Xu, E. K. Liu, Y. Du, G. J. Li, G. D. Liu, W. H. Wang, G. H. A. Wu, new spin gapless semiconductors family: Quaternary Heusler compounds. EPL (Europhysics Letters), 102(1), 17007. (2013)

21. A. Kundu, S. Ghosh, R. Banerjee, S. Ghosh, B. Sanyal, New quaternary half-metallic ferromagnets with large Curie temperatures. Scientific reports, 7(1), 1803. (2017)

22. I. Galanakis, P. H. Dederichs, N. Papanikolaou, Slater-Pauling behavior and origin of the half-metallicity of the full-Heusler alloys. Physical Review B, 66(17), 174429. (2002)

23. K. Özdoğan, E. Şaşığlu, I. Galanakis, Slater-Pauling behavior in LiMgPdSn-type multifunctional quaternary Heusler materials: Half-metallicity, spin-gapless and magnetic semiconductors. Journal of Applied Physics, 113(19), 193903. (2013) 


\section{Figure legends}

Figure 1. The three possible crystal structures of CoRhMnSn compound with their corresponding primitive unit cells.

Figure 2. Minimization of energy verses volume for CoRhMnSn.

Figure 3. Calculate band structures for CoRhMnSn compound at three different values of pressure:-16, 0 and $35 \mathrm{GPa}$.

Figure 4. Pressure effect on the edges of the spin minority bands in the CoRhMnSn compound.

Figure 5. The band structure for CoRhMnSn at 35GPa.

Figure 6. Variation of the total and partial magnetic moments as functions of the hydrostatic pressure.

Table1: The three possible atomic occupations of CoRhMnSn compound.

\begin{tabular}{|l|c|c|c|c|}
\hline & $4 \mathrm{a}(0,0,0)$ & $4 \mathrm{c}(1 / 4,1 / 4,1 / 4)$ & $4 \mathrm{~b}(1 / 2,1 / 2,1 / 2)$ & $4 \mathrm{~d}(3 / 4,3 / 4,3 / 4)$ \\
\hline Type I (Y1) & $\mathrm{Sn}$ & $\mathrm{Rh}$ & $\mathrm{Mn}$ & $\mathrm{Co}$ \\
\hline Type II (Y2) & $\mathrm{Sn}$ & $\mathrm{Mn}$ & $\mathrm{Rh}$ & $\mathrm{Co}$ \\
\hline Type III (Y3) & $\mathrm{Rh}$ & $\mathrm{Sn}$ & $\mathrm{Mn}$ & $\mathrm{Co}$ \\
\hline
\end{tabular}

Table 2. The calculated lowest total energy per unit cell, lattice constant, bulk modulus and its derivative for the $\mathrm{Y}_{1}-\mathrm{CoRhMnSn}$ crystal structure.

\begin{tabular}{|c|c|c|c|c|}
\hline & $E_{0}(\mathrm{eV})$ & $a_{0}(\AA)$ & $B_{0}(\mathrm{GPa})$ & $B^{\prime}$ \\
\hline Present work & -2390.879 & 6.154 & 188 & 5.14 \\
Expt. [10] & & 6.149 & & \\
Calc. [10] & & 6.164 & 177 & \\
\hline
\end{tabular}

\section{Acknowledgements}

Technical support from the LPTEA, LESI and LESIMS laboratories is gratefully acknowledged. 
Figures

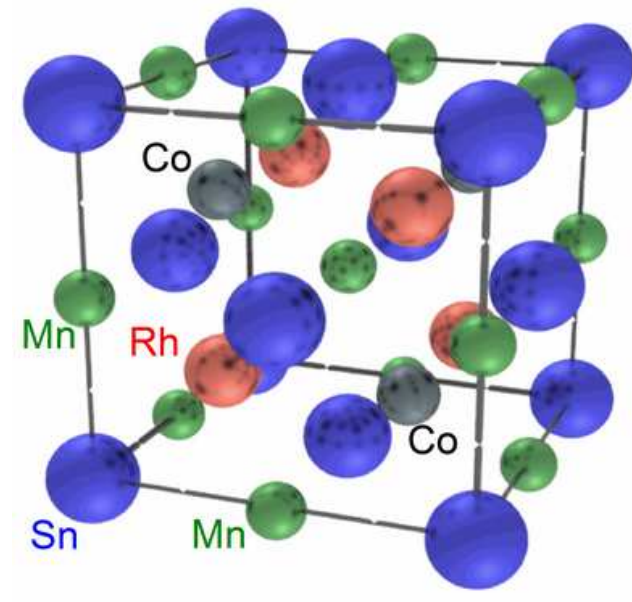

a) Type I

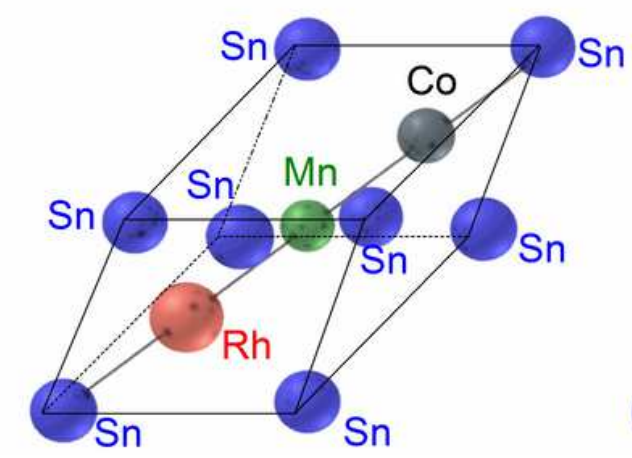

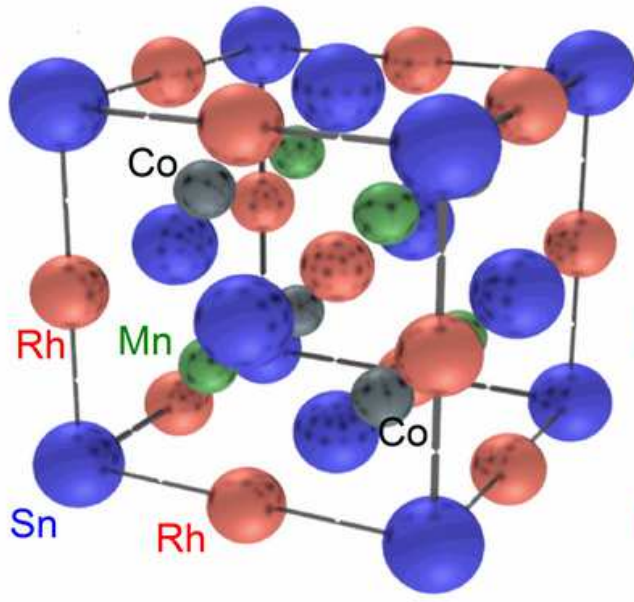

b) Type II

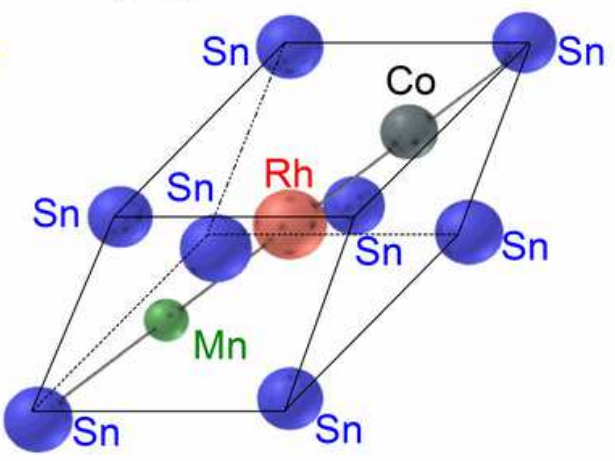

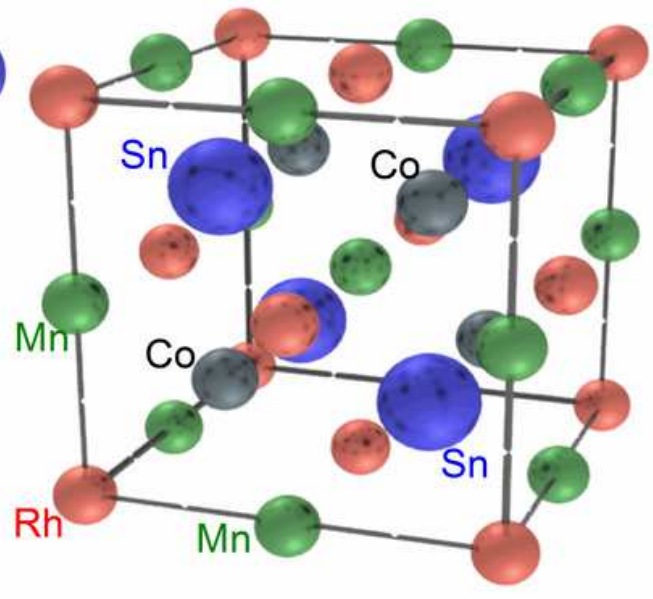

c) Type III

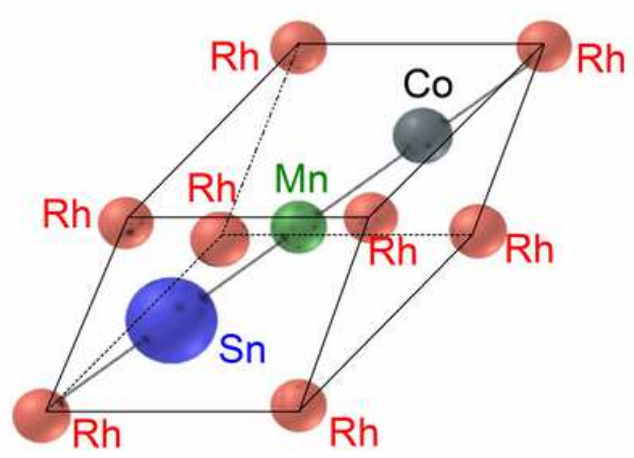

Figure 1

The three possible crystal structures of CoRhMnSn compound with their corresponding primitive unit cells. 


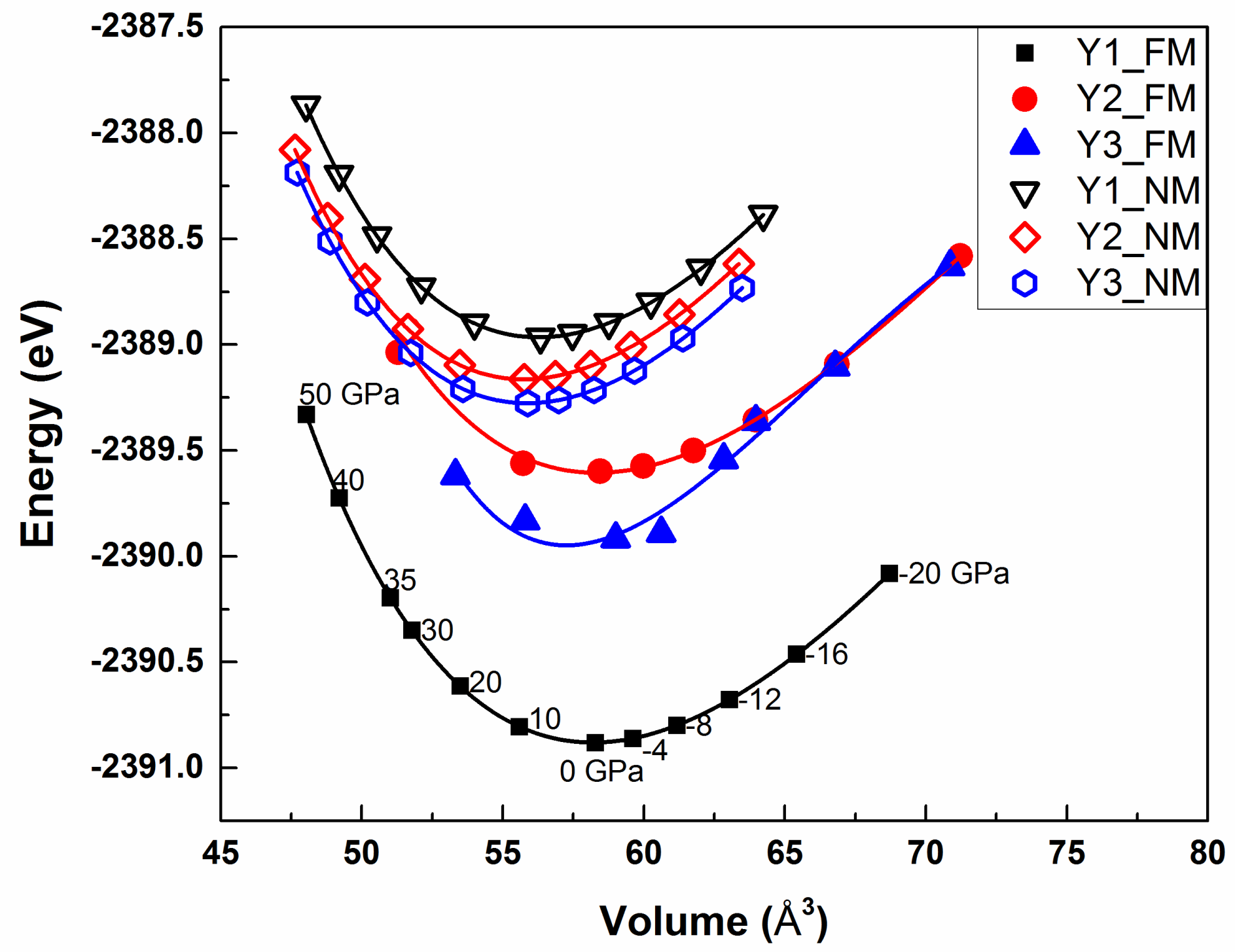

Figure 2

Minimization of energy verses volume for CoRhMnSn.
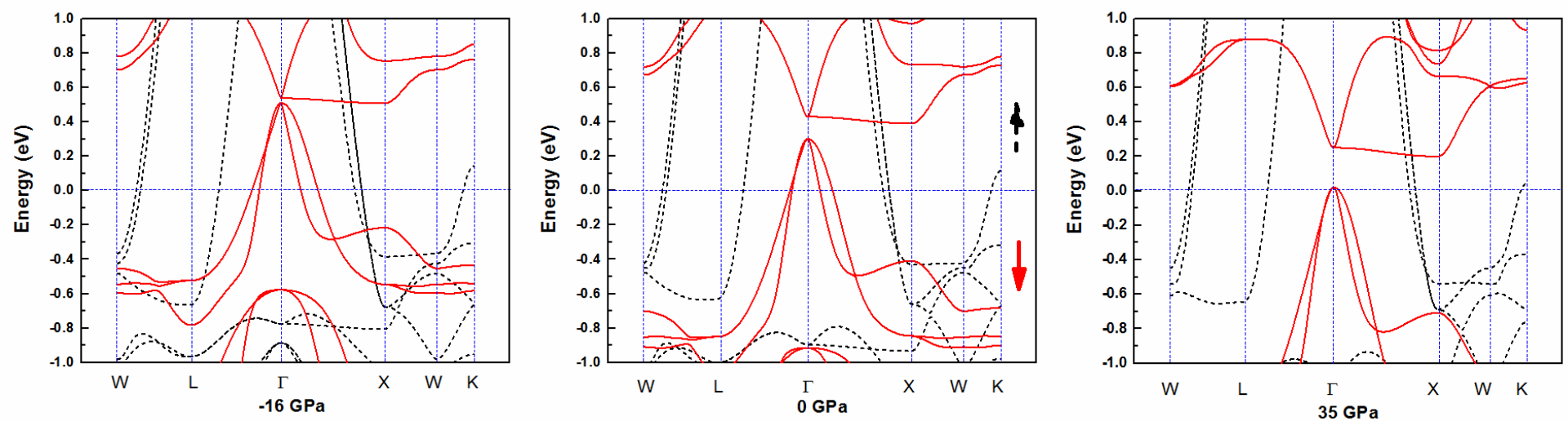

Figure 3 
Calculated band structures for CoRhMnSn compound at three different values of pressure:-16, 0 and 35GPa.

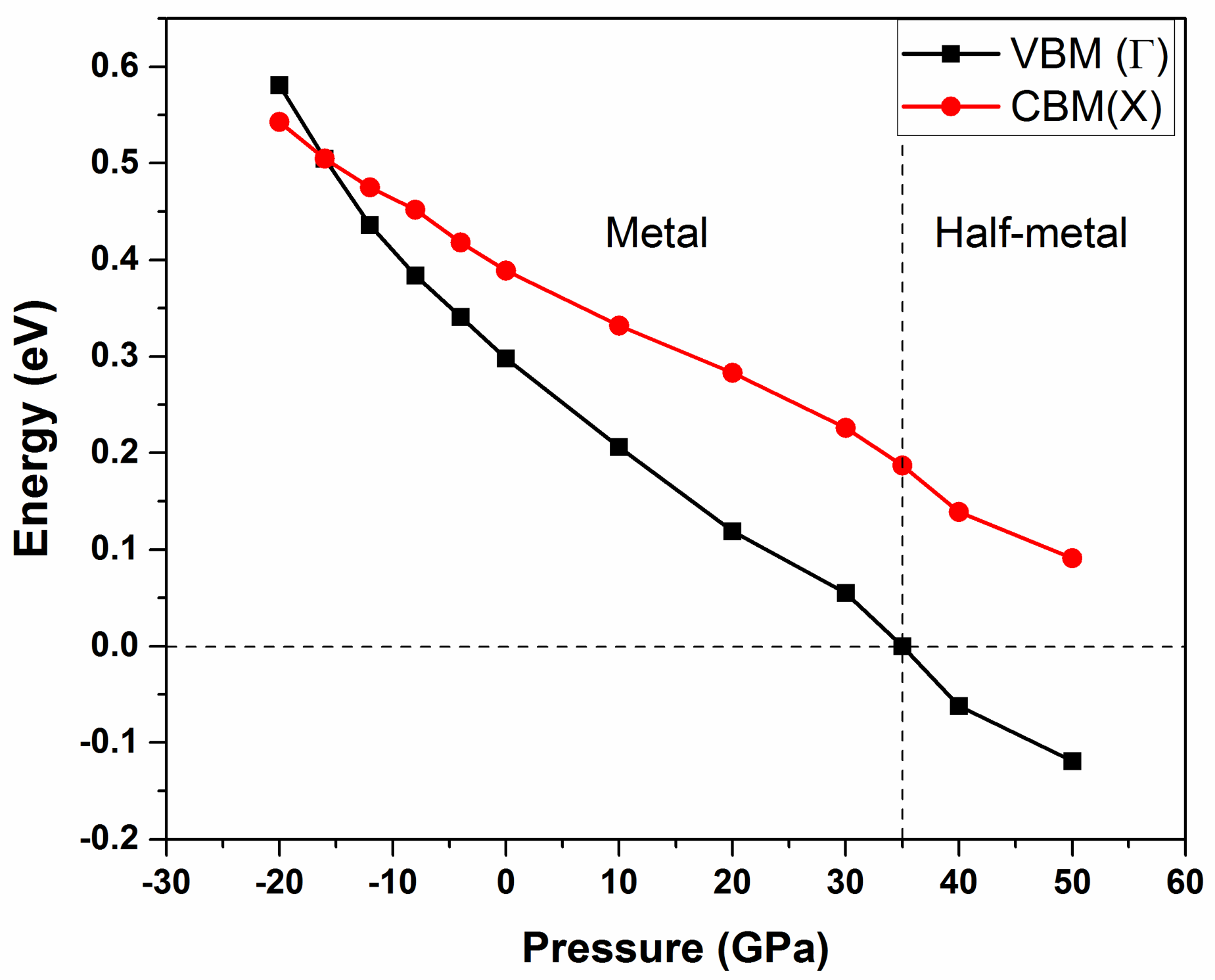

Figure 4

Pressure effect on the edges of the spin minority bands in the CoRhMnSn compound. 

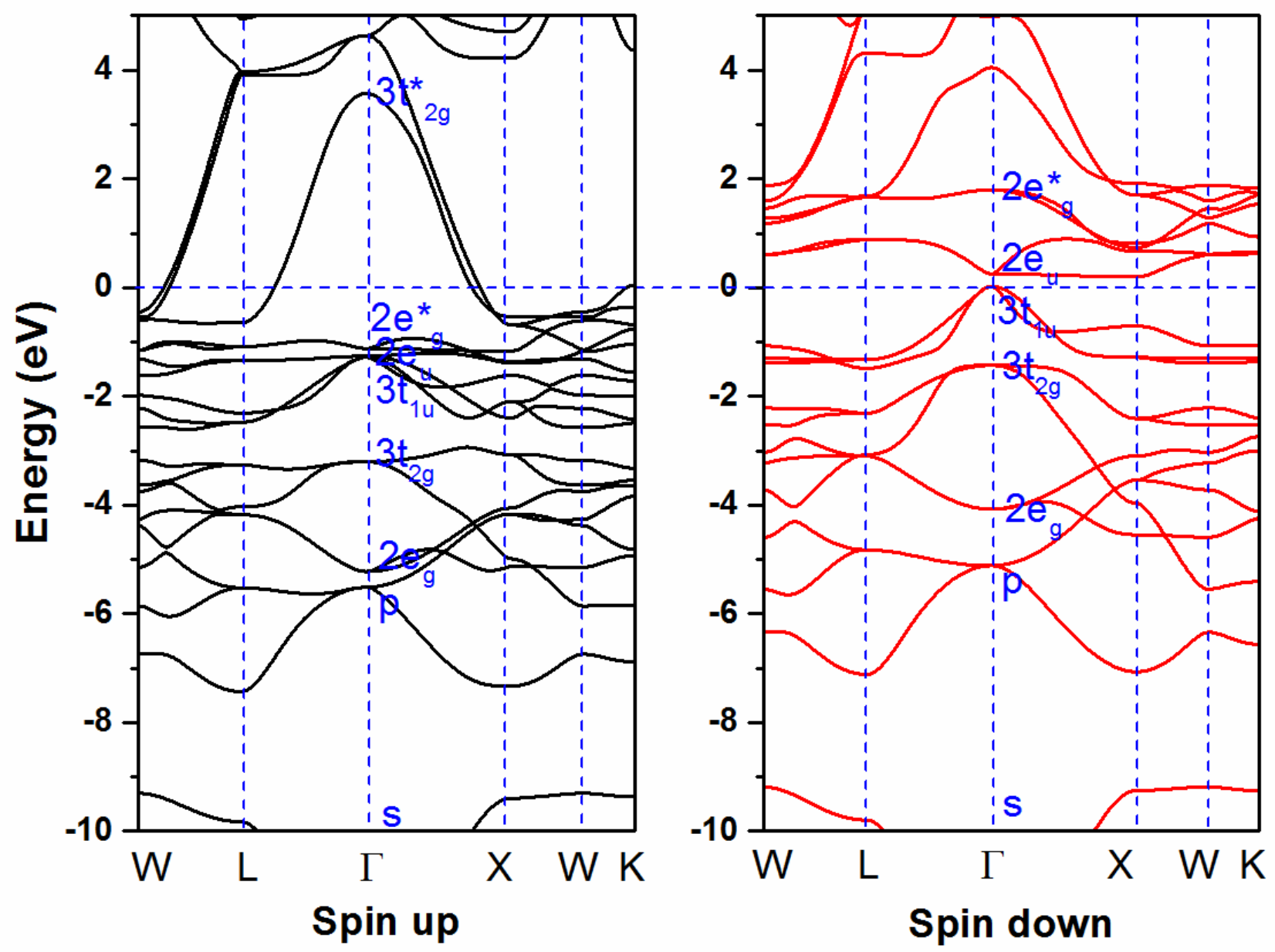

Figure 5

The band structure for CoRhMnSn at 35GPa. 


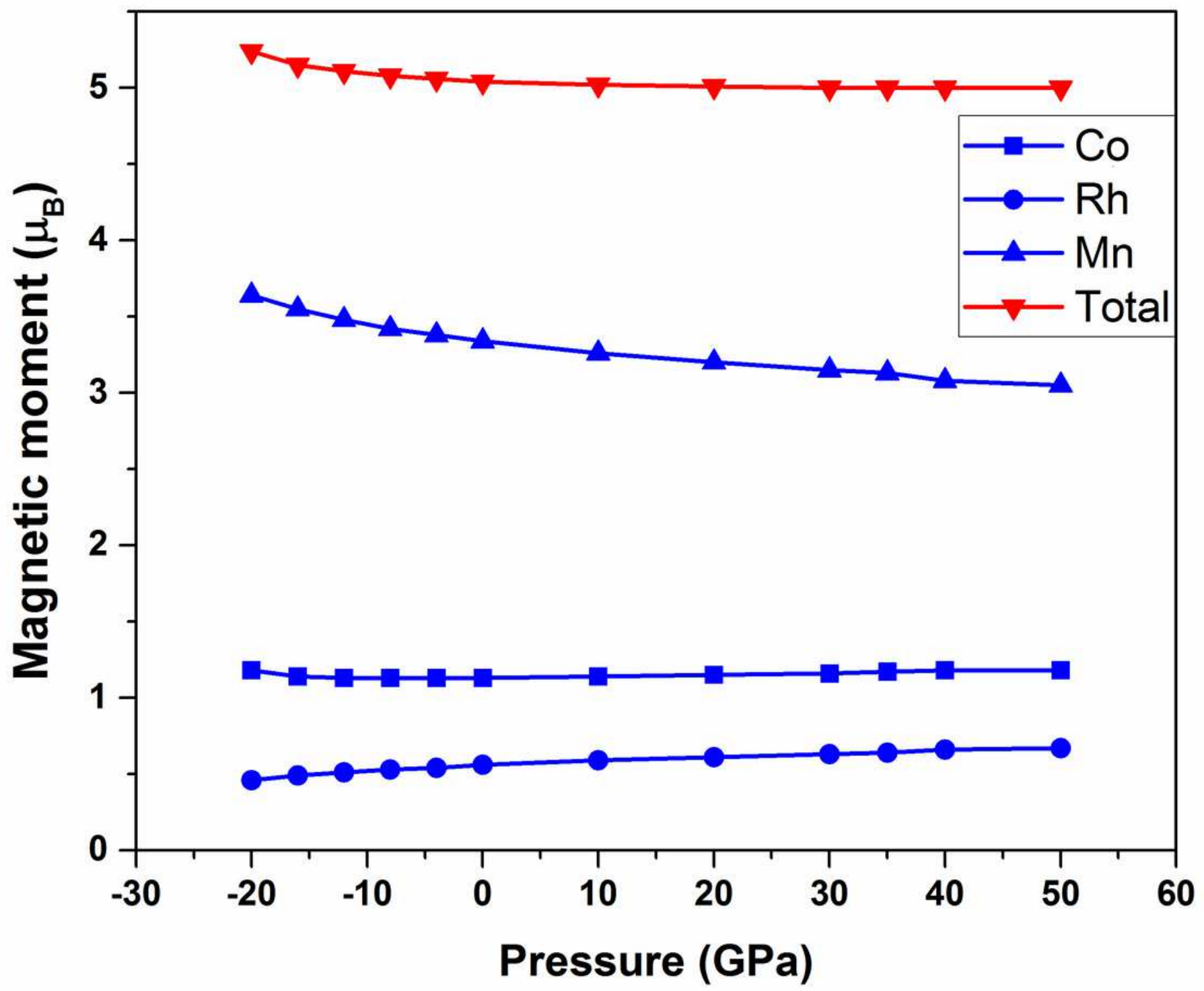

Figure 6

Variation of the total and partial magnetic moments as functions of the hydrostatic pressure. 